\title{
Intracellular cholesterol transport
}

\author{
Frederick R. Maxfield and Daniel Wüstner \\ Department of Biochemistry, Weill Medical College of Cornell University, New York, New York, USA
}

J. Clin. Invest. 110:891-898 (2002). doi:10.1172/JCI200216500.

The correct intracellular distribution of cholesterol among cellular membranes is essential for many biological functions of mammalian cells, including signal transduction and membrane traffic. Intracellular trafficking plays a major role in the proper disposition of internalized cholesterol and in the regulation of cholesterol efflux. Despite the importance of the transport and distribution of cholesterol within cells for normal physiology and in pathological conditions, many fundamental aspects of intracellular cholesterol movement are not well understood. For instance, the relative roles of vesicular and nonvesicular transport have not been fully determined, and the asymmetric distribution of cholesterol between the two leaflets of biological membranes is poorly characterized in many cases. Also, while it is clear that small, cholesterol-enriched microdomains (often referred to as rafts; see Simons and Ehehalt, this Perspective series, ref. 1) occur in many biological membranes, the composition, size, and dynamics of these microdomains remain uncertain. Here, we will discuss the current understanding of intracellular cholesterol transport.

\section{The intracellular distribution of cholesterol}

The membrane organelles of mammalian cells maintain distinct protein and lipid compositions that are essential for their proper function (Figure 1). In the biosynthetic secretory pathway, cholesterol is low in the endoplasmic reticulum (ER), but its level increases through the Golgi apparatus, with the highest levels in the plasma membrane (2). In the endocytic pathway, the endocytic recycling compartment (ERC), which contains recycling membrane proteins and lipids (3), also contains high levels of cholesterol (4). The cholesterol content of

\footnotetext{
Address correspondence to: Frederick R. Maxfield, Department of Biochemistry, Room E-215, Weill Medical College of Cornell University, 1300 York Avenue, New York, New York 10021, USA. Phone: (212) 747-6405; Fax: (212) 746-8875;

E-mail:frmaxfie@med.cornell.edu.

Conflict of interest: No conflict of interest has been declared. Nonstandard abbreviations used: endoplasmic reticulum (ER); endocytic recycling compartment (ERC); sterol response element-binding protein (SREBP); SREBP cleavage-activating protein (SCAP); detergent-resistant membrane (DRM); liquid-ordered (Lo); dehydroergosterol (DHE); ultraviolet (UV); horseradish peroxidase (HRP); steroidogenic acute regulatory protein (StAR); StAR-related lipid transfer (START); trans-Golgi network (TGN); Niemann-Pick C1 (NPC1).
}

late endosomes and lysosomes is not well documented, but under normal conditions it appears to be lower than in the ERC $(2,4)$. In polarized epithelial cells, the apical membrane is enriched in cholesterol and sphingolipids relative to the basolateral membrane (5).

Although the ER is the site of cholesterol synthesis, the concentration of cholesterol in the ER is very low, comprising only about $0.5-1 \%$ of cellular cholesterol (6) even though the surface area of the ER exceeds that of the plasma membrane in many cells. Many aspects of cholesterol regulation are under tight feedback control and are sensitive to the concentration of cholesterol in the ER. For example, an increase in the cholesterol content of the ER accelerates the degradation of 3-hydroxy-3-methylglutaryl-coenzyme A reductase, the key regulated enzyme for cholesterol synthesis (7). The sterol response element-binding protein (SREBP) cleavage-activating protein (SCAP) responds to reduction in cholesterol in the ER membrane by activating proteolytic cleavage and translocation of a fragment of SREBP into the nucleus, where it alters the transcription of several genes involved in cholesterol regulation (8). Thus, the sterol concentration in the ER membrane is crucial for cellular cholesterol homeostasis.

The cholesterol content of the Golgi apparatus is intermediate between those of the ER and the plasma membrane, but the measured cholesterol content of the Golgi depends on the method used for purification of these membranes (9). Electron microscopic filipin binding studies show an increased cholesterol content from the cis- to the trans-Golgi (7). It has been proposed that rafts enriched in sphingomyelin, glycosphingolipids, and cholesterol form in the Golgi apparatus and that these raft domains are selectively transported to the apical domain from the trans-Golgi $(5,10)$. The cholesterol in the ERC is similarly important for correct membrane trafficking. Reductions in cellular cholesterol have been shown to alter the recycling of glycosyl-phosphatidylinositol-anchored (GPIanchored) proteins (11).

The plasma membrane is estimated to contain about $60-80 \%$ of total cellular cholesterol (2), and it has been estimated that cholesterol is as much as $30-40 \%$ of the lipid molecules in the plasma membrane. Despite some uncertainty in these numbers, it is clear from all studies that the plasma membrane is highly enriched in cholesterol relative to other cellular membranes. 


\section{Cholesterol-rich microdomains}

Cholesterol and lipids are not homogeneously distributed within biological membrane bilayers. Cholesteroland sphingolipid-enriched microdomains, which are resistant to solubilization at low temperature by nonionic detergents such as Triton X-100, have been proposed to play an important role in cholesterol transport $(5,10,12)$. The properties of lipids in such detergentresistant membranes (DRMs) are similar to those in a liquid-ordered (Lo) phase that has been characterized in model membranes (13). Lo domains exhibit high lateral mobility of the lipids and tight packing in the hydrophobic core of the membrane. Rafts have not been observed directly by optical microscopy in living cells, presumably because their dimensions are below the resolution limit. The high lateral mobility within Lo domains and the small size of rafts in vivo (5) ensure that molecules will encounter the raft boundaries frequently and that individual molecules leave raftlike regions within seconds.

A high fraction of the plasma membrane of many types of cells is found in raftlike domains; about $70-80 \%$ of the surface area of several cell types has been shown to be resistant to solubilization by cold Triton X-100 (14). Fluorescence polarization studies also indicate that nearly half of the plasma membrane is in ordered domains at $37^{\circ} \mathrm{C}$, consistent with the high concentration of cholesterol and sphingolipids in the plasma membrane. In polarized epithelial cells, sphingolipids and cholesterol are especially enriched in the apical membrane domains, which may exist almost entirely in an ordered state (5).

The quantitative distribution of cholesterol between ordered and disordered domains in the plasma membrane is not known. Given the high overall concentration of cholesterol within the plasma membrane, only a small degree of local enrichment may be possible without disrupting the bilayer structure. Rather, the plasma membrane may be maintained at a composition where small changes in cholesterol content can cause large changes in membrane fluidity, as has been seen in model membranes (14).

Most likely, many types of membrane microdomains coexist in cells (14). Caveolae are one type of specialized, raftlike domain. They are associated with caveolins, and they have a characteristic flask shape with a diameter of about $60 \mathrm{~nm}$. In most cells, caveolae cover a few percent of the plasma membrane, so they represent a small fraction of the plasma membrane DRMs. Under certain circumstances, caveolae can pinch off from the plasma membrane (15), but under standard tissue culture conditions, plasma membrane caveolae do not exchange rapidly with internal pools of caveolin (16). Caveolins can bind cholesterol (17), but, as with other raftlike domains, the relative enrichment of cholesterol in caveolae is uncertain.

In addition to the lateral separation of membranes into various domains, the two leaflets of biological membranes have distinct compositions. Mechanisms for maintaining an asymmetric distribution of cholesterol are not well understood, but a likely mechanism is that the cholesterol distribution is largely determined by the other lipids in the membrane. In general, the transbilayer distribution of cholesterol in biological membranes other than the plasma membrane is not well characterized, and even in the plasma membrane there is some uncertainty. Some studies have indicated that sterol is predominantly in the outer leaflet of the plasma membrane (18), which would be consistent with a preferential association with sphingomyelin (13). However, several other studies have indicated that cholesterol is enriched in the cytoplasmic leaflet $(2,19)$, although the molecular basis for such an enrichment is unknown.

\section{Methods for studying cholesterol transport and distribution}

The uncertainty regarding cholesterol distribution in the inner and outer leaflets of the plasma membrane is one example of the more general difficulty of quantifying this and many other lipid species in intact biological membranes. In this case, since no direct measure of cholesterol is feasible, transbilayer distributions of cholesterol are determined by quenching emission from fluorescent sterols using membrane impermeant quenchers. This approach is not ideal, in part because there may be quantitative differences in the properties of the fluorescent cholesterol analogs as compared with cholesterol (see below).

Perhaps the most serious difficulty for the quantitative analysis of cholesterol in membranes is that, although cholesterol is poorly soluble in water, it can spontaneously desorb from membranes at an appreciable rate. Most often it will return to the same membrane, but it can also bind to whatever other hydrophobic binding sites are available. For this reason, a significant fraction of membrane cholesterol can redistribute among isolated organelles (20). In cells, soluble proteins can bind cholesterol, sometimes with high specificity and affinity $(21,22)$. Such proteins can also mediate transfer of cholesterol between membranes in vitro after cell disruption (22).

The transport and distribution of newly synthesized cholesterol can be determined by introducing ${ }^{3} \mathrm{H}$-acetate into living cells and measuring the amount of ${ }^{3} \mathrm{H}$ cholesterol in isolated membranes at different times. Radiolabeled cholesterol and cholesterol esters can be delivered by lipoproteins, and labeled cholesterol can also be delivered via specific cyclodextrin carriers, such as methyl- $\beta$-cyclodextrin. Total cholesterol can be measured by direct chemical methods such as gas chromatography-mass spectrometry or by indirect methods such as assays based on cholesterol oxidase $(2,23)$. In order for any of these methods of measuring cholesterol transport and distribution to be used, the various organelles of interest must be purified. It is generally quite difficult to obtain highly purified membrane fractions, so the possibility of effects from contaminating membranes must be considered. In addition, lengthy purification protocols may increase the risk of cholesterol transfer. These methods are most useful when organelles can be easily separated, as with the ER and 
the plasma membrane, but they can be very difficult to interpret when organelles such as endosomes and Golgi membranes are considered.

One of the most widely used tools for studying intracellular cholesterol distribution is the fluorescent detergent filipin, which binds selectively to cholesterol (and not to cholesterol esters) (24). Filipin staining can be used to detect cholesterol in various membrane organelles in intact cells. Although filipin is a relatively weak fluorophore, it is detected easily by cooled charge-coupled device cameras. Generally, filipin has been used for qualitative analyses of cholesterol distribution, since its fluorescence intensity is not necessarily linearly related to cholesterol content. For example, there are differences in the accessibility of cholesterol in different pools (25). Another limitation is that cholesterol might redistribute during long incubations with filipin. Thus, quantification of the intracellular cholesterol distribution from experiments using filipin is not possible, although cholesterol distributions observed with filipin are generally consistent with the distributions obtained by other methods. When filipin binds to cholesterol in membranes, it produces a characteristic bump in the membrane that can be seen in freeze-fracture micrographs. As with fluorescence studies, electron microscopy of filipin has been useful for qualitative analysis of cholesterol distribution.

Several fluorescent derivatives of cholesterol have been used in fluorescence microscopy studies. A major problem with fluorescent adducts of cholesterol is that the fluorophore can greatly change the properties of the cholesterol so that its distribution among cellular membranes is drastically altered (9). A few sterols are intrinsically fluorescent and have lateral and transbilayer distribution properties in membranes that are fairly similar to cholesterol. One of these, dehydroergosterol (DHE), is a naturally occurring sterol produced by yeast (26). Although it is a rapidly photobleached, weak fluorophore with emission in the near ultraviolet (UV), DHE can be incorporated into cellular membranes in sufficient concentrations to be observed with UV-sensitive charge-coupled device cameras $(4,9,27)$. DHE has also been used in a multiphoton imaging study (26). There are significant issues that need to be considered in validating DHE or other intrinsically fluorescent sterols as cholesterol analogs. DHE distributes similarly to cholesterol in cells $(4,9,27)$, but there are quantitative differences, for example, in its rate of desorption from model lipid membranes (28). Thus, there may be quantitative differences in the transport of DHE as compared with cholesterol. The fluorescence properties of DHE are also sensitive to the local environment, so relative fluorescence intensities in various membranes may not reflect concentration with complete accuracy. Nonetheless, these reagents offer the significant advantage that they can be used to directly observe sterol redistribution in living cells. DHE can be delivered to the plasma membrane via a methyl- $\beta$-cyclodextrin carrier in pulses as short as 1 minute, and redistribution can then be observed and quantified in pulse-chase studies $(4,27)$. The photobleaching of DHE can also be used to advantage by photobleaching one region of a cell and then measuring the rate at which the fluorescence returns as unbleached DHE exchanges into the bleached area $(4,9,27)$.

Some specialized techniques, such as the cholesterol oxidase assay (23), have been developed to quantify cholesterol in the plasma membrane. This assay will overestimate the amount of cholesterol on the plasma membrane if the enzyme gains access to intracellular compartments (e.g., by endocytosis in living cells or by membrane breakage) or if cholesterol moves to the plasma membrane during the assay. However, a modification of this approach, designed to minimize these problems (2), provides estimates of the fraction of cellular cholesterol at the plasma membrane (about 70\%) that are in general agreement with other methods. Another useful method to quantify cholesterol delivery to the plasma membrane is extraction by extracellular cyclodextrin $(29,30)$. This selective cholesterol acceptor can remove at least a pool of cholesterol from the membrane within a minute or so. In such efflux experiments, cells are equilibrated with ${ }^{3} \mathrm{H}$-cholesterol before adding extracellular cyclodextrins. The extraction of labeled cholesterol is typically biphasic, with a slow component of extraction requiring tens of minutes (30). It is likely that a major portion of the slow phase is delivery from internal organelles (4), but it is possible that there is also a slowly extractable pool in the plasma membrane (30). A slowly extractable plasma membrane pool would lead to uncertainty in estimates of the fraction of cholesterol at the plasma membrane by this method.

An enzymatic density shift method that has been used to determine whether proteins are in the same compartment has been adapted for determining the relative amount of ${ }^{3} \mathrm{H}$-cholesterol in organelles (4). When horseradish peroxidase (HRP) catalyzes peroxidation of diaminobenzidine, an insoluble polymer is formed within the organelle that creates a very large increase in its density. The organelles containing the HRP-catalyzed reaction product can be separated rapidly from the other cellular components by centrifugation. HRP can be delivered to endosomes by coupling to a protein such as transferrin. According to studies using this procedure, about $35 \%$ of cellular cholesterol is found in the early endosomes, including the ERC (4). HRP can also be targeted to organelles on the biosynthetic secretory pathway by expressing constructs encoding chimeric proteins containing HRP and an ER or Golgi protein (31). This method is subject to concerns about redistribution of cholesterol during the assay, but it provides a reasonably rapid and easy way to obtain relatively clean isolation of one organelle.

While many methods have been developed to measure cholesterol distribution in cells, all of them are subject to various degrees of uncertainty in their interpretation. It is therefore necessary to compare results obtained by several different methods in order to get a reliable analysis of intracellular cholesterol distribution. 
a

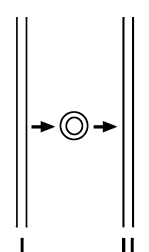

| b

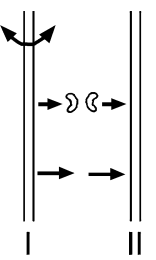

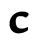

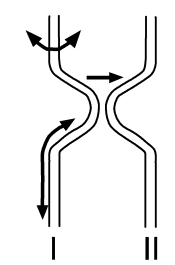

Figure 1

Basic mechanisms of cholesterol transport between two membranes. (a) Vesicular transport. This process requires ATP but does not require a change in the transversal distribution of cholesterol in the donor membrane. (b) Diffusion through the cytoplasm either bound to a carrier protein (upper arrows) or by free diffusion (lower arrows). Cholesterol in the donor membrane must desorb from the cytoplasmic leaflet, so the transbilayer distribution of cholesterol can affect this process. (c) Transport across membrane contacts. Adjacent donor and acceptor membranes come into close contact, resulting in cholesterol shuttling across the intermembrane space. This process requires cholesterol in the cytosolic leaflet of the donor membrane and may be facilitated by transport proteins.

\section{Mechanisms for intracellular sterol transport}

Another great challenge in studying intracellular cholesterol transport is that several fundamentally different mechanisms to move sterols operate simultaneously in live cells (Figure 1). The combined action of these pathways can make it hard to obtain a clear overall understanding of cholesterol transport.

Cholesterol can be incorporated into transport vesicles or tubules that carry membrane constituents from one organelle to another. Pharmacological inhibition can be used to test the importance of various membrane trafficking steps. For example, the involvement of the Golgi apparatus can be tested using brefeldin A, which causes Golgi membranes to become fused into the ER (32). Microtubule-mediated movement of vesicles can be tested using nocodazole or other agents that disrupt microtubules (32). Expression of dominant inhibitory proteins can also be used to assess the role of vesicle traffic. For example, export of cholesterol or DHE from the ERC (Figure 2) requires ATP and can be blocked by expression of a mutant form of an ERCassociated protein, Rme-1 (4).

Since cholesterol can desorb from membranes at a significant rate and cells have many possible cholesterol carriers in the cytoplasm, carrier-mediated diffusion can play an important role in transport of cholesterol among cellular membranes (Figure 1). Nonvesicular transport is important for sterol transport from the plasma membrane to the ERC (4) (Figure 2) and for delivery of cholesterol to the inner mitochondrial membrane in steroidogenic cells (22). The cholesterol carriers could consist of a large number of cytosolic proteins each with low affinity and specificity for cholesterol. Alternatively, the carriers could be specialized cholesterol transport proteins. A family of high-affinity lipid and sterol carriers has been identified of which one of the prototypes is the steroidogenic acute regulatory protein (StAR/StarD1) (21), whose lipid- or sterol-binding domains are called StAR-related lipid transfer (START) domains. StAR/StarD1 has been implicated

in the delivery of cholesterol to mitochondrial cytochrome P450 in steroidogenic cells (ref. 22; see also Jefcoate, this Perspective series, ref. 33). There are now several family members known, some of which have been shown to bind cholesterol with high affinity. The expression of one of these, StarD4, is regulated by cellular sterol levels (34). Interestingly, another family member, MLN64, has a transmembrane domain that localizes it to late endosomes and a START domain that binds cholesterol (35). It is not known whether the main function of these START domain proteins is sterol and lipid transport or whether they are primarily regulatory proteins.

Diffusible sterol-binding proteins provide a rapid mechanism for shuttling cholesterol among membranes, but the basis of specificity in membrane targeting is not known. One possibility is that these factors are targeted to certain compartments by binding to proteins or lipids that are enriched in those compartments. While it seems likely that specific targeting mechanisms exist, an alternate model holds that diffusible carriers distribute sterol among all possible target membranes, with the relative enrichment in various organelles determined by the ability of the membranes to serve as sterol acceptors. In support of the latter model, the initial rate of cholesterol transfer among organelles isolated from fibroblasts appears to be determined largely by the characteristics of the acceptor membrane (20). Moreover, DHE on a cyclodextrin carrier has been found to be delivered preferentially to the ERC not only in living cells, but even in permeabilized, formaldehyde-fixed cells (4), consistent with the idea that this process is energy-independent and arises from the intrinsic properties of the target membranes.

Another means to facilitate transport from one membrane to another is to have a close contact between the two membranes. For example, in many cell types, part of the ER is in close proximity to the plasma membrane (36), an arrangement that could facilitate rapid exchange of cholesterol between these two membranes, perhaps with the assistance of transfer proteins. Similarly, three-dimensional reconstructions of the Golgi apparatus have revealed extensive areas of close apposition between the trans-Golgi and ER (37). The cholesterol-esterifying enzyme ACAT is enriched in the parts of the ER near to the ERC and the trans-Golgi (38), perhaps allowing efficient delivery of cholesterol to ACAT from these cholesterol-rich membranes.

Caveolae and caveolin have been proposed to play an important role in intracellular cholesterol transport. In cultured fibroblasts, reduction in caveolin expression by antisense DNA suppresses cholesterol efflux, whereas transfection with caveolin cDNA stimulates this process (39). The mechanisms by which caveolin could affect cholesterol transport remain uncertain, although caveolin can bind cholesterol directly (17). It has been proposed that caveolin forms a complex with chaperone proteins that deliver cholesterol from the ER to the plasma membrane, bypassing the Golgi apparatus (40). Palmitoylation of caveolin-1 may be required for rapid $\left(t_{1 / 2}=10\right.$ minutes) transfer of cholesterol from the ER 
to caveolae via this chaperone complex (40). However, some studies have failed to find evidence that this cholesterol chaperone complex participates in surface delivery of newly synthesized cholesterol (32), and several caveats need to be resolved about the contribution of caveolin to cholesterol transport. Thus, at least in cultured cells, the rate of caveolin flux between the cell interior and the plasma membrane is typically quite low (16). Moreover, mice lacking expression of both caveolin-1 and caveolin-2 $(41,42)$ appear to be normal with regard to their cholesterol levels and regulation, although a full study of cholesterol transport in these mice has not yet been reported.

A more indirect role for caveolins and other related proteins has been proposed in which increased accumulation of caveolin-1 in the ER promotes its interaction with newly formed lipid droplets (43). It is suggested that the membrane topology of caveolin, which has two cytoplasmic domains flanking a central region that penetrates but does not cross the bilayer, might allow it to associate preferentially with the membrane deformations that appear during the formation of a lipid droplet. Interestingly, a family of plant proteins associated with lipid droplets, the oleosins, have a topology similar to caveolins. Hence, caveolins and related proteins may alter the membrane bilayer structure, promoting membrane curvature, and perhaps facilitating the transbilayer redistribution of cholesterol.

\section{General intracellular transport pathways}

Transport to and from the ER. Since the ER is the site of cholesterol synthesis but maintains a low steady-state level of cholesterol, efficient transport mechanisms must exist to export cholesterol from the ER (Figure 2). Cholesterol export from the ER requires metabolic energy (2). As measured by extracting cholesterol upon arrival at the plasma membrane with cyclodextrin, the kinetics of its transport appears to be biphasic (29), with a first phase, corresponding to about half of the total, reaching the plasma membrane in 60 minutes, and the remaining material being released in a slow phase over 5-6 hours (29).

The vesicle-mediated protein secretory pathway through the Golgi apparatus could, in principle, provide a parallel route for newly synthesized cholesterol to the plasma membrane. Biochemical studies have demonstrated that this is not a major pathway of cholesterol transport from the ER to the plasma membrane. Brefeldin A treatment can quantitatively suppress cell surface transport of vesicular stomatitis virus G protein without blocking cholesterol transport (44). Still, although this vesicular route is not a major pathway of cholesterol export, a recent study found that some cholesterol exported from the ER becomes confined to DRMs before reaching the plasma membrane and that transport of cholesterol to the plasma membrane can be partly inhibited by brefeldin A (32). The passage of some cholesterol through the Golgi apparatus might be important for raft-dependent sorting in the trans-Golgi network (TGN) of polarized epithelia (5).
In addition, energy depletion or treatments that interfere with vesicle traffic have been found to reduce the esterification of $\beta$-VLDL-derived cholesterol in macrophages at a stage after release of cholesterol from late endosomes and lysosomes (45). However, treatment of cells with bacterial sphingomyelinase, which releases cholesterol from the plasma membrane, leads to ATPindependent cholesterol esterification and is accompanied by an ATP-independent vesiculation of the plasma membrane $(45,46)$. It thus appears that both vesicular and nonvesicular mechanisms operate throughout the cholesterol transport process.

Export from late endosomes and lysosomes. Cholesterol esters in LDL and other lipoproteins are hydrolyzed to free cholesterol in late endosomes and lysosomes. The cholesterol is exported from the lysosomes by a mechanism that is not fully characterized (Figure 2). Transport from lysosomes to the ER or the plasma membrane can be inhibited by progesterone or hydrophobic amines, such as U18666A, imipramine, sphinganine, and stearylamine (2), but this inhibition does not seem to be at the step of export from the late endosomes and lysosomes, since LDL-derived cholesterol appears at the plasma membrane with similar kinetics in treated and untreated cells (47). After efflux from the late endosomes and lysosomes, cholesterol can be delivered to various other organelles. It has been estimated that in fibroblasts about $30 \%$ of the LDL-derived cholesterol is delivered to the ER without transit through the plasma membrane $(2,29)$.

Niemann-Pick C disease is an inherited recessive disorder characterized by accumulation of cholesterol and other lipids in organelles that share many but not all characteristics of late endosomes $(7,48)$. The Niemann-Pick C1 (NPC1) protein is a multiplemembrane-spanning protein that does not transport

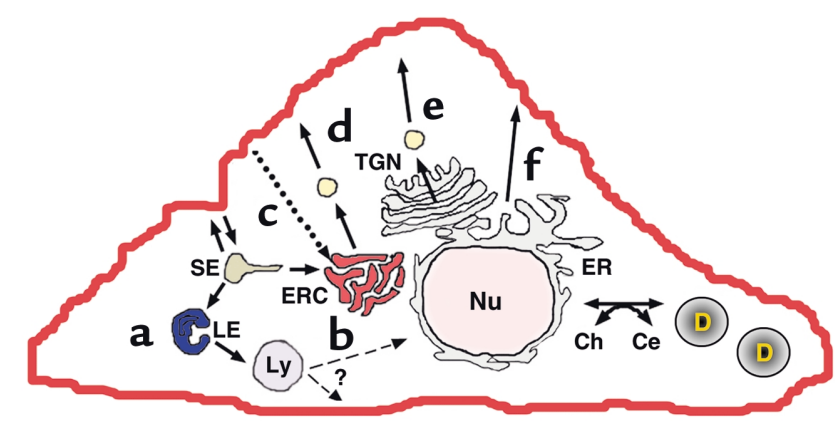

\section{Figure 2}

Cholesterol transport in nonpolarized cells. LDL carrying cholesterol and CE (esterified cholesterol) is transported (a) from sorting endosomes (SE) to late endosomes (LE) and lysosomes (Ly), from which cholesterol can efflux and reach the plasma membrane or the ER, where it gets esterified (b). Efflux from LE and Ly is poorly characterized, as indicated by dashed lines. Cholesterol can move from the plasma membrane to the ERC by a nonvesicular, ATP-independent process (c). In contrast, recycling of cholesterol occurs almost exclusively in vesicles also carrying other recycling markers (d). De novo synthesized cholesterol is mostly transported from the ER directly to the plasma membrane, bypassing the Golgi apparatus (f), but some follows the biosynthetic secretory pathway from the ER to the TGN (e). Excess cholesterol ( $\mathrm{Ch}$ ) in the ER becomes esterified (CE) and stored in cytoplasmic lipid droplets (D). 


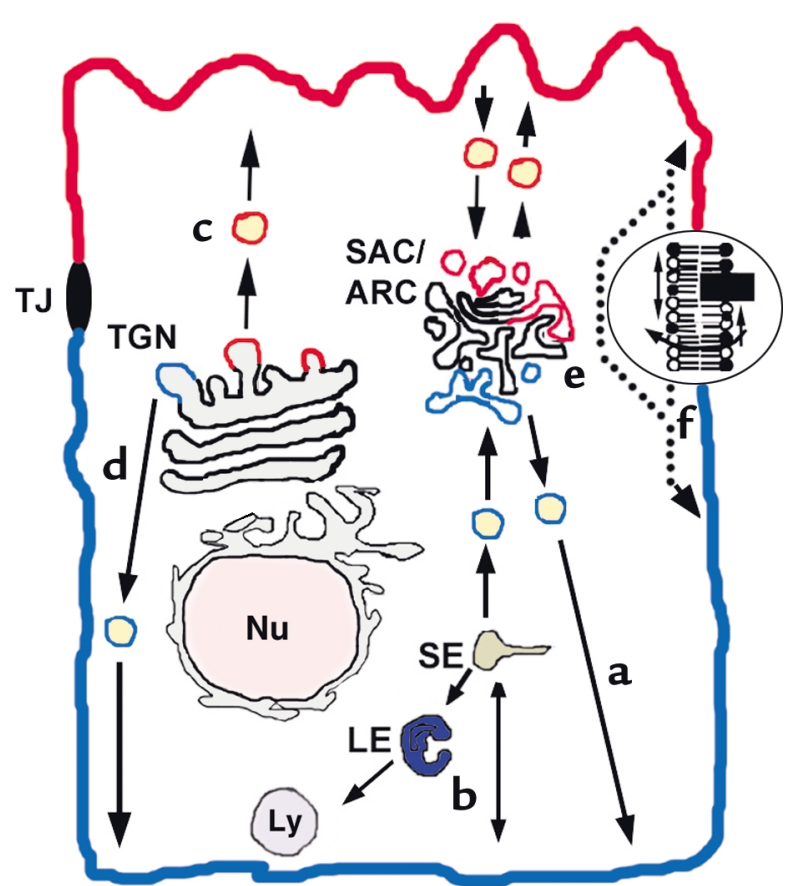

Figure 3

Cholesterol transport in polarized cells. Polarized cells form distinct apical (red) and basolateral (blue) membrane compartments, which are separated by tight junctions (TJ). Proteins and lipids are sorted along the biosynthetic and endocytic pathways indicated by blue (basolateral) and red (apical) vesicles. Plasma membrane cholesterol is transported in vesicles between the basolateral and apical membrane via a subapical compartment or apical recycling compartment (SAC/ARC). Recycling to the basolateral membrane can also occur from this compartment (a). LDL cholesterol has the same fate as in nonpolarized cells $(\mathbf{b})$. A fraction of de novo synthesized cholesterol is transported along the biosynthetic pathway as in nonpolarized cells. In the TGN, cholesterol might form microdomains or rafts along with sphingolipids that segregate from the remaining TGN membrane and carry apically destined proteins and lipids to the apical membrane shown in red (c). Basolaterally destined vesicles (blue) bud off of the TGN but should contain less cholesterol (d). Plasma membrane cholesterol can shuttle rapidly between the plasma membrane domains by nonvesicular transport. This process involves fast transbilayer migration of cholesterol to circumvent the lateral diffusion barrier created by the $\mathrm{TJ}$ in the exoplasmic leaflet of the plasma membrane. Transport through the cytoplasm bound to a protein carrier (e), and/or diffusion along the inner monolayer $(\mathbf{f})$ result in rapid exchange of cholesterol between the apical and basolateral plasma membrane domain. CE formation occurs as in nonpolarized cells but is omitted for clarity.

cholesterol directly but that can facilitate the transbilayer transport of some hydrophobic molecules (49). It is unclear at what step or steps the NPC1 protein acts, but, as with progesterone or hydrophobic amines, it appears that initial export from late endosomes is not affected; cholesterol taken into the cells as an LDL-associated ester exits the late endosomes and appears at the plasma membrane as free cholesterol at the same rate in wild-type and NPC1 mutant cells $(47,50)$. Nevertheless, cholesterol ultimately accumulates in storage organelles that resemble late endosomes and are enriched in lysobisphosphatidic acid (48) and other lipids (7). The basis for this cholesterol and lipid accumulation in the NPC1 mutants or in cells treated with hydrophobic amines remains uncertain.

Efflux from the plasma membrane. There has been considerable progress in the past few years in understanding the mechanisms of cholesterol efflux to extracellular acceptors (see Tall et al., this Perspective series, ref. 51). The protein that is defective in Tangier disease, ABCA1, plays a key, but indirect, role in cholesterol efflux to HDL acceptors (12). It appears that the specific role of ABCA1 is to facilitate phospholipid transfer to apoA-I. Cholesterol can then be effluxed to the phospholipid-loaded apoA-I by a mechanism that does not require ABCA1 (52). The role of membrane specializations in cholesterol efflux is an active area of investigation. Caveolae have been described as important sites for efflux, but it is not clear whether these have been distinguished from other, more abundant types of raftlike membranes (12). Interestingly, cells overexpressing ABCA1 carry plasma membrane protrusions (53) that may play a role in facilitating phospholipid and cholesterol transfer.

\section{Intracellular transport of cholesterol in specialized cells}

Hepatic cholesterol transport. Chylomicron remnants and other types of lipoproteins are taken up by hepatocytes using a variety of receptors (54). After hydrolysis of cholesterol esters, free cholesterol is released into the cell where it can be shuttled directly to the canalicular membrane for biliary secretion, used for synthesis of bile salts, or re-esterified and used for assembly of VLDLs in the ER (55). Assembled VLDL is secreted at the basolateral membrane of hepatocytes and carries cholesterol to peripheral tissues (56).

The intracellular trafficking pathways of cholesterol in hepatocytes and other epithelia are poorly defined. In addition to the pathways available in nonpolarized cells, epithelial cells maintain distinct lipid and protein compositions in their apical and their basolateral plasma membranes, requiring specialized sorting in the biosynthetic secretory pathway and in the endocytic recycling pathways $(3,57)$ (Figure 3 ). Rapid, nonvesicular exchange of DHE between the apical and basolateral plasma membrane domains has been observed in the polarized hepatoma cell line HepG2, which forms an apical vacuole resembling the biliary canaliculus (27). DHE is transported in vesicles to a subapical compartment or apical recycling compartment (SAC/ARC), suggesting that cholesterol derived from the plasma membrane of hepatocytic cells has rapid access to various intracellular compartments.

Cholesterol transport in macrophages. Since loading of cholesterol esters in cytoplasmic droplets of macrophages is an early step in formation of atherosclerotic lesions, the intracellular transport of cholesterol in these cells is of great interest (58). Many of the transport pathways are similar to those found in other cells. In macrophages, the intracellular fate of cholesterol derived from lipoproteins depends on the mechanism underlying its internalization. LDL is 
internalized by the LDL receptor and delivered to late endosomes, where cholesterol esters are hydrolyzed; homeostatic downregulation of LDL receptors limits the total cholesterol delivery from this source, and cholesterol ester lipid droplets do not accumulate in these cells. In contrast, cholesterol delivered via $\beta$-VLDL, which also binds to the LDL receptor, becomes esterified by ACAT and stored in cytoplasmic droplets, perhaps because a greater load of cholesterol is delivered by the larger $\beta$-VLDL particles. Oxidized or acetylated LDLs enter via scavenger receptors and also stimulate formation of cholesterol ester droplets, although the oxidized LDL is much less effective in activating this process (59). LDL aggregated and retained on the ECM does not become internalized by receptor-mediated endocytosis but remains in prolonged extracellular contact with macrophages; in this case, delivery of the associated cholesterol precedes internalization and degradation of the proteins (60). This interaction, which resembles the situation in atherosclerotic lesions, also leads to significant stimulation of cholesterol esterification (58).

The extent of cholesterol esterification by macrophages depends in a nonlinear fashion on the amount of cholesterol loading (61), such that much more esterification occurs once a threshold loading value is exceeded. The basis for this nonlinear response is not fully understood, but one component of it may be more efficient delivery of cholesterol to ACAT in the ER when free cholesterol is above the threshold. A similar nonlinear relationship between plasma membrane cholesterol elevation and levels of cholesterol in the ER has been found in fibroblasts (6), and such a relationship would be consistent with the effects seen in macrophages as cholesterol is elevated.

Cholesterol transport in steroidogenic cells. Cholesterol is the precursor for the synthesis of steroid hormones in mitochondria of gonadal and adrenal tissue. The synthesis is initiated by conversion of cholesterol to pregnenolone via the $\mathrm{C} 27$ cholesterol side chain cleavage cytochrome $\mathrm{P} 450$, located in the inner mitochondrial membrane. Delivery of cholesterol is rate-limiting to this process (21). Cholesterol destined for steroid hormone synthesis is mainly derived from the plasma membrane and from lipid droplets. How cholesterol reaches the outer surface of mitochondria is not yet defined, but nonvesicular transport mechanisms via carrier proteins or via membrane contacts between mitochondria and lipid droplets are likely to be important $(62,63)$. It is generally assumed that the rate-limiting step in steroidogenesis is the translocation of cholesterol from the outer to the inner mitochondrial membrane, and several proteins have been proposed to mediate this transport step, including the peripheral-type benzodiazepine receptor, which binds cholesterol and other compounds known to stimulate steroidogenesis (64). StAR/StarD1 is thought to act selectively at the outer mitochondria surface to mediate import of cholesterol (refs. 65, 66; see also Jefcoate, this Perspective series, ref. 33 , for an alternative view), but the exact mechanism of StAR-induced cholesterol import into mitochondria is not known.

\section{Concluding remarks}

The intracellular distribution of unesterified cholesterol among various cellular organelles is determined by multiple mechanisms including transbilayer flipping, stabilization in membrane microdomains by interactions with lipids (and perhaps proteins), enrichment or exclusion from transport vesicles as they form, and binding to diffusible carrier proteins in the cytoplasm and within intracellular organelles. Some of these transport mechanisms involve passive movement of cholesterol down a free-energy gradient, but others require metabolic energy to overcome kinetic barriers or to move cholesterol up a gradient. Cholesterol transport from one organelle to another can be accomplished by combinations of these mechanisms, which operate in parallel within the cell. The mechanisms and regulation of the resulting, complex pathways are still only partially understood. Further progress will require better understanding of transport pathways, including a kinetic and morphological description of the movement of cholesterol, as well as a more complete description of the proteins that control the key events in the various pathways.

\section{Acknowledgments}

We are grateful to Timothy McGraw and Mingming Hao for reading the manuscript. This work was supported by grants from the NIH (DK-27083) and the Ara Parseghian Medical Research Foundation. D. Wüstner is supported by a postdoctoral fellowship from the Charles H. Revson Foundation. Space limitations precluded referencing a number of important studies. Additional references may be found in the suggested reading list available at www.jci.org/cgi/content/ full/110/07/891/DC1.

1. Simons, K., and Ehehalt, R. 2002. Cholesterol, lipid rafts, and disease. J. Clin. Invest. 110:597-603. doi:10.1172/JCI200216390.

2. Liscum, L., and Munn, N.J. 1999. Intracellular cholesterol transport. Biochim. Biophys. Acta. 1438:19-37.

3. Mukherjee, S., Ghosh, R.N., and Maxfield, F.R. 1997. Endocytosis. Physiol. Rev. 77:759-803.

4. Hao, M., et al. 2002. Vesicular and non-vesicular sterol transport in living cells. The endocytic recycling compartment is a major sterol storage organelle. J. Biol. Chem. 277:609-617.

5. Simons, K., and Ikonen, E. 1997. Functional rafts in cell membranes. Nature. 387:569-572.

6. Lange, Y., Ye, J., Rigney, M., and Steck, T.L. 1999. Regulation of endoplasmic reticulum cholesterol by plasma membrane cholesterol. J. Lipid. Res. 40:2264-2270.

7. Blanchette-Mackie, E. 2000. Intracellular cholesterol trafficking: role of the NPC1 protein. Biochim. Biophys. Acta. 1486:171-183.

8. Brown, M., and Goldstein, J. 1999. A proteolytic pathway that controls the cholesterol content of membranes, cells, and blood. Proc. Natl. Acad. Sci. USA. 96:11041-11048.

9. Mukherjee, S., Zha, X., Tabas, I., and Maxfield, F. 1998. Cholesterol distribution in living cells: fluorescence imaging using dehydroergosterol as a fluorescent cholesterol analog. Biophys. J. 75:1915-1925.

10. Ikonen, E. 2001. Roles of lipid rafts in membrane transport. Curr. Opin. Cell Biol. 13:470-477.

11. Mayor, S., Sabharanjak, S., and Maxfield, F. 1998. Cholesterol-dependent retention of GPI-anchored proteins in endosomes. EMBOJ. 17:4626-4638.

12. Fielding, C., and Fielding, P. 2001. Cellular cholesterol efflux. Biochim. Biophys. Acta. 1533:175-189.

13. Brown, D., and London, E. 2000. Structure and function of sphingolipidand cholesterol-rich membrane rafts. J. Biol. Chem. 275:17221-17224.

14. Maxfield, F.R. 2002. Plasma membrane microdomains. Curr. Opin. Cell Biol. 14:483-487.

15. Pelkmans, L., Puntener, D., and Helenius, A. 2002. Local actin polymerization and dynamin recruitment in SV40-induced internalization of caveolae. Science. 296:535-539. 
16. Thomsen, P., Roepstorff, K., Stahlhut, M., van Deurs, B. 2002. Caveolae are highly immobile plasma membrane microdomains, which are not involved in constitutive endocytic trafficking. Mol. Biol. Cell. 13:238-250.

17. Ikonen, E., and Parton, R. 2000. Caveolins and cellular cholesterol balance. Traffic. 1:212-217.

18. Boesze-Battaglia, K., Clayton, S., and Schimmel, R. 1996. Cholesterol redistribution within human platelet plasma membrane: evidence for a stimulus-dependent event. Biochemistry. 35:6664-6673.

19. Schroeder, F., et al. 2001. Recent advances in membrane microdomains: rafts, caveolae, and intracellular cholesterol trafficking. Exp. Biol. Med. 226:873-890.

20. Frolov, A., Woodford, J.K., Murphy, E.J., Billheimer, J.T., and Schroeder, F. 1996. Spontaneous and protein-mediated sterol transfer between intracellular membranes. J. Biol. Chem. 271:16075-16083.

21. Stocco, D. 2001. StAR protein and the regulation of steroid hormone biosynthesis. Annu. Rev. Physiol. 63:193-213.

22. Strauss, J., et al. 1999. The steroidogenic acute regulatory protein (StAR): a window into the complexities of intracellular cholesterol trafficking. Recent Prog. Horm. Res. 54:369-394.

23. Lange, Y. 1992. Tracking cell cholesterol with cholesterol oxidase. J. Lipid Res. 33:315-321.

24. Schroeder, F., Holland, J., and Bieber, L. 1971. Fluorometric evidence for the binding of cholesterol to the filipin complex. J. Antibiot. (Tokyo). 24:846-849.

25. Steer, C.J., Bisher, M., Blumenthal, R., and Steven, A.C. 1984. Detection of membrane cholesterol by filipin in isolated rat liver coated vesicles is dependent upon removal of the clathrin coat. J. Cell Biol. 99:315-319.

26. Frolov, A., et al. 2000. High density lipoprotein-mediated cholesterol uptake and targeting to lipid droplets in intact L-cell fibroblasts. A single- and multiphoton fluorescence approach. J. Biol. Chem. 275:12769-12780

27. Wüstner, D., Herrmann, A., Hao, M., and Maxfield, F.R. 2002. Rapid nonvesicular transport of sterol between the plasma membrane domains of polarized hepatic cells. J. Biol. Chem. 277:30325-30336.

28. Ohvo-Rekila, H., Akerlund, B., and Slotte, J. 2000. Cyclodextrin-catalyzed extraction of fluorescent sterols from monolayer membranes and small unilamellar vesicles. Chem. Phys. Lipids. 105:167-178.

29. Neufeld, E.B., et al. 1996. Intracellular trafficking of cholesterol monitored with a cyclodextrin. J. Biol. Chem. 271:21604-21613.

30. Haynes, M., Phillips, M., and Rothblat, G. 2000. Efflux of cholesterol from different cellular pools. Biochemistry. 39:4508-4517.

31. Connolly, C., Futter, C., Gibson, A., Hopkins, C., and Cutler, D. 1994. Transport into and out of the Golgi complex studied by transfecting cells with cDNAs encoding horseradish peroxidase. J. Cell Biol. 127:641-652.

32. Heino, S., et al. 2000. Dissecting the role of the Golgi complex and lipid rafts in biosynthetic transport of cholesterol to the cell surface. Proc. Natl. Acad. Sci. USA. 97:8375-8380.

33. Jefcoate, C. 2002. High-flux mitochondrial cholesterol trafficking, a specialized function of the adrenal cortex. J. Clin. Invest. 110:881-890. doi:10.1172/JCI200216771.

34. Soccio, R., et al. 2002. The cholesterol-regulated StarD4 gene encodes a StAR-related lipid transfer protein with two closely related homologues, StarD5 and StarD6. Proc. Natl. Acad. Sci. USA. 99:6943-6948.

35. Alpy, F., et al. 2001. The steroidogenic acute regulatory protein homolog MLN64, a late endosomal cholesterol-binding protein. J. Biol. Chem. 276:4261-4269.

36. Putney, J. 1999. “Kissin' cousins”: intimate plasma membrane-ER interactions underlie capacitative calcium entry. Cell. 99:5-8.

37. Ladinsky, M., Mastronarde, D., McIntosh, J., Howell, K., and Staehelin, L. 1999. Golgi structure in three dimensions: functional insights from the normal rat kidney cell. J. Cell Biol. 144:1135-1149.

38. Khelef, N., et al. 2000. Enrichment of acyl coenzyme A:cholesterol O-acyltransferase near trans-golgi network and endocytic recycling compartment. Arterioscler. Thromb. Vasc. Biol. 20:1769-1776.

39. Fielding, C., Bist, A., and Fielding, P. 1999. Intracellular cholesterol transport in synchronized human skin fibroblasts. Biochemistry. 38:2506-2513.

40. Uittenbogaard, A., and Smart, E. 2000. Palmitoylation of caveolin-1 is required for cholesterol binding, chaperone complex formation, and rapid transport of cholesterol to caveolae. J. Biol. Chem. 275:25595-25599.
41. Drab, M., et al. 2001. Loss of caveolae, vascular dysfunction, and pulmonary defects in caveolin-1 gene-disrupted mice. Science. 293:2449-2452.

42. Razani, B., et al. 2002. Caveolin-1-deficient mice are lean, resistant to diet-induced obesity, and show hypertriglyceridemia with adipocyte abnormalities. J. Biol. Chem. 277:8635-8647.

43. Ostermeyer, A., et al. 2001. Accumulation of caveolin in the endoplasmic reticulum redirects the protein to lipid storage droplets. J. Cell Biol. 152:1071-1078.

44. Urbani, L., and Simoni, R.D. 1990. Cholesterol and vesicular stomatitis virus $\mathrm{G}$ protein take separate routes from the endoplasmic reticulum to the plasma membrane. J. Biol. Chem. 265:1919-1923.

45. Skiba, P.J., Zha, X., Maxfield, F.R., Schissel, S.L., and Tabas, I. 1996. The distal pathway of lipoprotein-induced cholesterol esterification, but not sphingomyelinase-induced cholesterol esterification, is energy-dependent. J. Biol. Chem. 271:13392-13400.

46. Zha, X., et al. 1998. Sphingomyelinase treatment induces ATP-independent endocytosis. J. Cell Biol. 140:39-47.

47. Lange, Y., Ye, J., Rigney, M., and Steck, T. 2000. Cholesterol movement in Niemann-Pick type C cells and in cells treated with amphiphiles. J. Biol. Chem. 275:17468-17475.

48. Kobayashi, T., et al. 1999. Late endosomal membranes rich in lysobisphosphatidic acid regulate cholesterol transport. Nat. Cell Biol. 1:113-118.

49. Davies, J., Chen, F., and Ioannou, Y. 2000. Transmembrane molecular pump activity of Niemann-Pick C1 protein. Science. 290:2295-2298.

50. Cruz, J., Sugii, S., Yu, C., and Chang, T. 2000. Role of Niemann-Pick type $\mathrm{C} 1$ protein in intracellular trafficking of low density lipoprotein-derived cholesterol. J. Biol. Chem. 275:4013-4021.

51. Tall, A.R., Costet, P., and Wang, N. 2002. Regulation and mechanisms of macrophage cholesterol efflux. J. Clin. Invest. 110:899-904. doi:10.1172/JCI200216391.

52. Wang, N., Silver, D., Thiele, C., and Tall, A. 2001. ATP-binding cassette transporter $\mathrm{A} 1$ (ABCA1) functions as a cholesterol efflux regulatory protein. J. Biol. Chem. 276:23742-23747.

53. Wang, N., Silver, D., Costet, P., and Tall, A. 2000. Specific binding of ApoA-I, enhanced cholesterol efflux, and altered plasma membrane morphology in cells expressing ABC1. J. Biol. Chem. 275:33053-33058.

54. Havel, R. 1995. Chylomicron remnants: hepatic receptors and metabolism. Curr. Opin. Lipidol. 6:312-316.

55. Vance, J., and Vance, D. 1990. Lipoprotein assembly and secretion by hepatocytes. Annu. Rev. Nutr. 10:337-356.

56. Kang, S., and Davis, R. 2000. Cholesterol and hepatic lipoprotein assembly and secretion. Biochim. Biophys. Acta. 1529:223-230.

57. Zegers, M., and Hoekstra, D. 1998. Mechanisms and functional features of polarized membrane traffic in epithelial and hepatic cells. Biochem. J. 336:257-269.

58. Tabas, I. 2000. Cholesterol and phospholipid metabolism in macrophages. Biochim. Biophys. Acta. 1529:164-174.

59. Tabas, I. 1999. Nonoxidative modifications of lipoproteins in atherogenesis. Annu. Rev. Nutr. 19:123-139.

60. Sakr, S., et al. 2001. The uptake and degradation of matrix-bound lipoproteins by macrophages require an intact actin cytoskeleton, Rho family GTPases, and myosin ATPase activity. J. Biol. Chem. 276:37649-37658.

61. Xu, X.X., and Tabas, I. 1991. Lipoproteins activate acyl-coenzyme A:cholesterol acyltransferase in macrophages only after cellular cholesterol pools are expanded to a critical threshold level. J. Biol. Chem. 266:17040-17048.

62. Kallen, C., et al. 1998. Steroidogenic acute regulatory protein (StAR) is a sterol transfer protein. J. Biol. Chem. 273:26285-26288.

63. Hall, P., and Almahbobi, G. 1997. Roles of microfilaments and intermediate filaments in adrenal steroidogenesis. Microsc. Res. Tech. 36:463-479.

64. Papadopoulos, V., et al. 1997. Peripheral benzodiazepine receptor in cholesterol transport and steroidogenesis. Steroids. 62:21-28.

65. Arakane, F., et al. 1998. The mechanism of action of steroidogenic acute regulatory protein (StAR). StAR acts on the outside of mitochondria to stimulate steroidogenesis. J. Biol. Chem. 273:16339-16345.

66. Bose, H., Lingappa, V., and Miller, W. 2002. Rapid regulation of steroidogenesis by mitochondrial protein import. Nature. 417:87-91. 